\title{
An overview of the Phalaenopsis orchid genome through BAC end sequence analysis
}

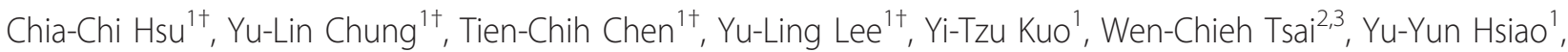 \\ Yun-Wen Chen ${ }^{1}$, Wen-Luan $\mathrm{Wu}^{1,2,3^{*}}$, Hong-Hwa Chen ${ }^{1,2,3^{*}}$
}

\begin{abstract}
Background: Phalaenopsis orchids are popular floral crops, and development of new cultivars is economically important to floricultural industries worldwide. Analysis of orchid genes could facilitate orchid improvement. Bacterial artificial chromosome (BAC) end sequences (BESs) can provide the first glimpses into the sequence composition of a novel genome and can yield molecular markers for use in genetic mapping and breeding.
\end{abstract}

Results: We used two BAC libraries (constructed using the BamHI and Hindlll restriction enzymes) of Phalaenopsis equestris to generate pair-end sequences from 2,920 BAC clones (71.4\% and 28.6\% from the BamHI and Hindlll libraries, respectively), at a success rate of $95.7 \%$. A total of 5,535 BESs were generated, representing $4.5 \mathrm{Mb}$, or about $0.3 \%$ of the Phalaenopsis genome. The trimmed sequences ranged from 123 to 1,397 base pairs (bp) in size, with an average edited read length of $821 \mathrm{bp}$. When these BESs were subjected to sequence homology searches, it was found that $641(11.6 \%)$ were predicted to represent protein-encoding regions, whereas 1,272 (23.0\%) contained repetitive DNA. Most of the repetitive DNA sequences were gypsy- and copia-like retrotransposons (41.9\% and 12.8\%, respectively), whereas only $10.8 \%$ were DNA transposons. Further, 950 potential simple sequence repeats (SSRs) were discovered. Dinucleotides were the most abundant repeat motifs; AT/TA dimer repeats were the most frequent SSRs, representing 253 (26.6\%) of all identified SSRs. Microsynteny analysis revealed that more BESs mapped to the whole-genome sequences of poplar than to those of grape or Arabidopsis, and even fewer mapped to the rice genome. This work will facilitate analysis of the Phalaenopsis genome, and will help clarify similarities and differences in genome composition between orchids and other plant species.

Conclusion: Using BES analysis, we obtained an overview of the Phalaenopsis genome in terms of gene abundance, the presence of repetitive DNA and SSR markers, and the extent of microsynteny with other plant species. This work provides a basis for future physical mapping of the Phalaenopsis genome and advances our knowledge thereof.

\section{Background}

The family Orchidaceae, which contains at least 25,000 species, is one of the largest families of flowering plants [1]. As with all other living organisms, present-day orchids have evolved from ancestral forms as a result of selection pressure and adaptation. Orchids show a wide diversity of epiphytic and terrestrial growth forms, and these plants have successfully colonized almost every habitat on earth. The factors promoting the richness of

\footnotetext{
*Correspondence: wenluan2@mail.ncku.edu.tw; hhchen@mail.ncku.edu.tw + Contributed equally

'Department of Life Sciences, National Cheng Kung University, Tainan 701, Taiwan

Full list of author information is available at the end of the article
}

orchid species may include specific interactions between orchid flowers and pollinators [2], sequential and rapid interplay between drift and natural selection [3], obligate orchid-mycorrhizal interactions [4], and epiphytism. The latter mode is the growth form of more than $70 \%$ of all orchids [5], which comprise approximately two-thirds of the epiphytic flora of the world.

Expansion of diversity may have taken place more quickly in the orchid family than in most other flowering plant families, which had already started to diversify in the mid-Cretaceous [6]. The time at which orchids originated is disputed, but it has been suggested to be 80-40 million years ago (Mya) (thus in the late Cretaceous to late Eocene) [7]. Recently, the Orchidaceae were
C Biomed Central

() 2011 Hsu et al; licensee BioMed Central Ltd. This is an Open Access article distributed under the terms of the Creative Commons Attribution License (http://creativecommons.org/licenses/by/2.0), which permits unrestricted use, distribution, and reproduction in any medium, provided the original work is properly cited. 
dated using an amber fossil of an orchid pollinia on the back of the pollinator, a stingless bee [8]. The most recent common ancestor of extant orchids is believed to have lived in the late Cretaceous (76-84 Mya) [8]. Perhaps the only general statement that can be made about the origin of orchids is that most extant groups are probably very young.

Orchids are known for the diversity of their specialized reproductive and ecological strategies. Formation of the labellum and gynostemium (a fused structure of the androecium and gynoecium) to facilitate pollination has been thoroughly documented, and the co-evolution of orchid flowers and pollinators thereof is well understood $[9,10]$. The successful evolutionary progress of orchids may be explained by the packaging of mature pollen grains as pollinia, the pollination-based regulation of ovary/ovule development, the synchronized timing of micro- and mega-gametogenesis for effective fertilization, and the release of thousands or millions of immature embryos (endosperm-free seeds) in a mature capsule [11]. However, despite the unique aspects of developmental reproductive biology and the specialized pollination and ecological strategies of orchids, relatively few molecular studies have focused on orchids compared to other species-rich plant families [12].

The genomic sequence resources for orchids are limited. A number of studies have used Sanger sequencing to develop expressed sequence tag (EST) resources for orchids [13-15]. These works have highlighted the usefulness of cDNA sequencing in the discovery of candidate genes for orchid floral development [16,17], floral scent production $[14,18]$, and flowering time determination [19], in the absence of a full genomic sequence. However, we do not yet have a comprehensive description of all genes that are expressed in orchids.

Hybrids of the genus Phalaenopsis are among the toptraded blooming potted plants worldwide. Because the plants possess favorable commercial traits, such as numerous spikes and branches, along with many colorful flowers, $P$. equestris is often used as a parent for breeding in its native Taiwan. $P$. equestris is a diploid plant with 38 chromosomes $(2 \mathrm{n}=2 \mathrm{x})$ that are small and uniform in size $(<2 \mu \mathrm{m}$ long) [20]. The plant has an estimated haploid genome size of 1,600 $\mathrm{Mb}$ (3.37 pg/diploid genome), which is relatively small compared to those of other members of the genus Phalaenopsis [21]. Public databases of floral bud ESTs from $P$. equestris and P. bellina have been developed and analyzed [14,17]; they provide valuable opportunities for researchers to directly access genes of interest [16-18] and to identify molecular markers useful in marker-assisted breeding programs or cultivar identification (unpublished data). However, we still lack basic information on the sequence, organization, and structure of the Phalaenopsis genome.
One efficient and viable strategy for gaining insight into the sequence content and complexity of the Phalaenopsis genome is afforded by the construction of bacterial artificial chromosome (BAC) libraries and end-sequencing of randomly selected BAC clones. Such BAC end sequences (BESs) can be used as a primary scaffold for genome shotgun-sequence assembly and to generate comparative physical maps [22]. Analysis of BES data can provide an overview of the sequence composition of a novel genome, yielding information on gene density, and the presence of potential transposable elements (TEs) and microsatellites [23-26]. In addition, BESs can identify molecular markers that may be used for genomic mapping and cloning, and in phylogenetic analysis. Even for the rice genome, which has been fully sequenced, the Oryza Map Alignment Project (OMAP) constructed deep-coverage large-insert BAC libraries from 11 wild and 1 cultivated African Oryza species (O. glaberrima); clones from these 12 BAC libraries were next fingerprinted and end-sequenced. The resulting data were used to construct physical maps of the Oryza species to permit studies on evolution, genome organization, domestication, gene regulatory networks, and efforts toward crop improvement $[27,28]$. However, such work has not yet been performed in orchids.

In the present study, we analyzed 5,535 BESs of two genomic BAC libraries of $P$. equestris, focusing on simple sequence repeat (SSR) or microsatellite content, repeat element composition, GC content, and proteinencoding regions. The annotated BESs reported herein offer the first detailed insights into the sequence composition of the $P$. equestris genome, and should be a useful resource for future molecular marker development.

\section{Results and Discussion \\ $B A C$ end sequencing}

Two large-insert bacterial artificial chromosome (BAC) libraries were used for end-sequencing in the present study. One library, constructed from a partial HindIII digest of $P$. equestris genomic DNA, consisted of 100,992 clones with an average insert size of $100 \mathrm{~kb}$. The other library, constructed from a partial BamHI digest, consisted of 33,428 clones with an average insert size of $111 \mathrm{~kb}$. The two libraries represent approximately 8.4 equivalents of the wild-type Phalaenopsis haploid genome.

DNA samples extracted from 2,920 BAC clones (71.4\% and 28.6\% from the BamHI and HindIII libraries, respectively) were sequenced from both ends using Applied Biosystems (ABI) Big Dye terminator chemistry followed by analysis on ABI 3730 machines. The success rate was $95.7 \%$. After ambiguous, vector, and mitochondrial DNA sequences were omitted, 5,535 high-quality BESs remained; these included 5,360 paired-end reads (Table 1). The BESs ranged in size from 123 to $1,397 \mathrm{bp}$ 
Table 1 Statistical analysis of Phalaenopsis equestris bacterial artificial chromosome (BAC) end sequences (BESs)

\begin{tabular}{lc}
\hline Total number of BESs & 5,535 \\
No. of paired BESs & 5,360 \\
No. of non-paired BESs & 175 \\
Total length (bp) & $4,544,250$ \\
Minimum length (bp) & 123 \\
Maximum length (bp) & 1,397 \\
Average length (bp) & 821 \\
GC content & $35.95 \%$ \\
Sequence composition & \\
$\quad$ Potential transposable elements (\%) & $1,272(23.0)$ \\
$\quad$ Simple sequence repeats (\%) & $950(17.2)$ \\
$\quad$ Protein coding regions (\%) & $641(11.6)$ \\
$\quad$ Chloroplast sequences (\%) & $29(0.5)$ \\
$\quad$ Unknown genomic sequences (\%) & $2,643(47.7)$ \\
\hline
\end{tabular}

(average, $821 \mathrm{bp}$ ) and corresponded to a total length of $4,544,250 \mathrm{bp}$, which is equivalent to $0.3 \%$ of the $P$. equestris genome (Table 1). The 5,535 BESs could be assembled into 340 contigs (average coverage $=2.99$ ) and 4,518 singletons (data not shown). In terms of readlength distribution, 800-899 bp and 900-999 bp were the most abundant categories, accounting for 1,567 (28\%) and 1,684 (30\%) of all BESs, respectively (Figure 1). The GC content was $35.95 \%$; this is comparable to the $34.09 \%$ previously estimated by buoyant density analysis of the genomic DNA of P. amabilis BLUME (Sarcanthinae; Vandeae) [29], indicating that the Phalaenopsis genome is AT-rich. Buoyant density analysis has also been used to study Brassica maculate R. BR. (Oncidiinae, Vandeae), Cattleya schombocattleya LINDL. (Epidendrinae, Epidendrae), and Cymbidium pumilum SWARTZ cv. "Gareth Latangor" (Cymbidiinae, Vandeae), which had GC contents of $32.05 \%, 34.09 \%$, and $32.05 \%$, respectively [29].

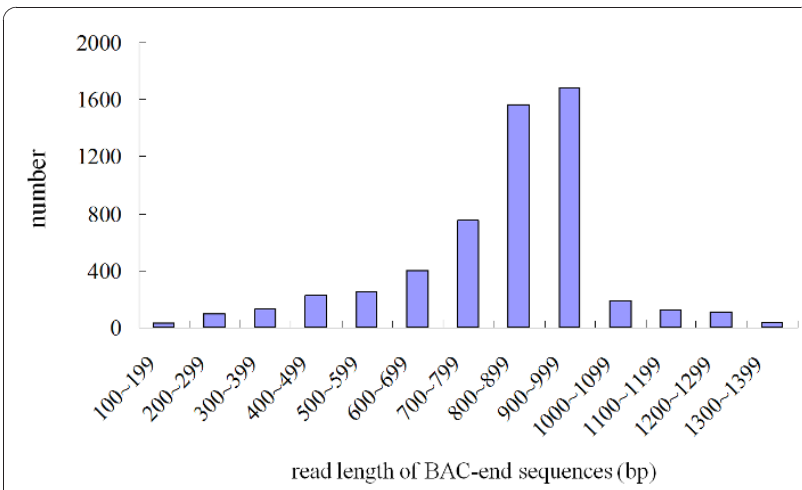

Figure 1 Size distribution of Phalaenopsis equestris bacterial artificial chromosome (BAC) end sequences. The trimmed sequences ranged from 123 to $1,397 \mathrm{bp}$ in length and had an average edited read length of $821 \mathrm{bp}$.
Thus, the available evidence suggests that most orchids have AT-rich genomes. All BES sequences generated herein have been deposited in GenBank under accession numbers HN176659-HN182163.

\section{Database sequence searches}

The $P$. equestris BESs were subjected to sequence homology analysis using the RepBase and TIGR plant repeat databases, and RepeatMasker and BLAST were employed to predict repeat sequences and potential TEs, respectively. A total of $1,272 \mathrm{BESs}$ (23.0\% of total) were found to harbor putative TEs and repeats. The BESs were also RepeatMasked and compared to data in the NCBI non-redundant protein databases. A total of 641 (11.6\%) were found to contain protein-coding sequences; of these, $29 \mathrm{BESs}(0.5 \%$ of the total) contained putative chloroplast DNA-encoded genes (Table 1).

\section{Analysis of repetitive DNA in the BESs}

The large genome size of $P$. equestris $(1,600 \mathrm{~kb})$ implies that the content of repetitive DNA could be high, rendering the genome more similar to that of maize than rice. The 29 BESs containing apparent chloroplast sequences were removed from analysis, and the remaining 5,506 BESs were screened for repetitive DNA sequences, using RepeatMasker and the TIGR plant repeat database. As for other eukaryotic genomes, that of Phalaenopsis was found to contain a significant proportion of repeat sequences and potential TEs; 1,272 BESs (23\% of the total) contained such TEs (Table 1). This percentage was higher than that of apple (20.9\%) [25], but lower than that of Citrus clementina (25.4\%) [30], carrot (28.3\%) [26], or Musa acuminata (36.6\%) [24].

Among the 1,272 BESs containing potential TEs, more showed sequence homology to Class I retrotransposons (963 BESs, 75.7\%) than to Class II DNA transposons (137 BESs, 10.8\%), suggesting a 7:1 Class I:Class II ratio in the genome (Table 2). The Class I retrotransposons could be further classified into Ty1/copia (163, $12.8 \%$ ) and Ty3/gypsy (533, 41.9\%) long-terminal-repeat (LTR) retrotransposons; LINE $(95,7.5 \%)$ and SINE $(0,0.0 \%)$ non-LTR retrotransposons; and other unclassified retrotransposons $(172,13.5 \%)$ (Table 2). Clearly, the LTR retrotransposons outnumbered those of the nonLTR form $(696,54.7 \%$ vs. 95, 7.5\%); the number of unclassified retrotransposons $(172,13.5 \%)$. The next most abundant DNA repeat type was Class II DNA transposons, which included Ac/Ds (12, 0.9\%), En/Spm (67, 5.3\%), Mutator (15, 1.2\%), Tourist, Harbinger, Helitron, Mariner $(10,0.8 \%)$, and other unclassified transposons $(33,2.6 \%)$. In total, $1,100 \mathrm{BESs}$ were found to contain Class I retrotransposons and Class II DNA transposons. The other identified repeat sequences 
Table 2 Number of bacterial artificial chromosome (BAC) end sequences (BESs) containing repetitive DNA

\begin{tabular}{llc}
\hline Class, subclass, group & $\begin{array}{l}\text { No. of } \\
\text { BESs }\end{array}$ & $\begin{array}{c}\text { \% of BESs with } \\
\text { repetitive DNA }\end{array}$ \\
\hline Class I retrotransposons & 963 & 75.7 \\
Ty1-copia & 163 & 12.8 \\
Ty3-gypsy & 533 & 41.9 \\
LINE & 95 & 7.5 \\
SINE & 0 & 0.0 \\
Unclassified retrotransposons & 172 & 13.5 \\
Class II DNA transposons & 137 & 10.8 \\
Ac/Ds & 12 & 0.9 \\
CACTA, En/Spm & 67 & 5.3 \\
Mutator (MULE) & 15 & 1.2 \\
Tourist/Harbinger/Helitron/ & 10 & 0.8 \\
Mariner & & \\
Unclassified Transposons & 33 & 2.6 \\
Miniature inverted-repeat & 2 & 0.2 \\
transposable elements & & 1.5 \\
Centromere & 19 & 2.6 \\
rRNA & 33 & 9.3 \\
Unclassified & 118 & 100 \\
\hline Total & 1272 & \\
& &
\end{tabular}

included miniature inverted-repeat transposable elements (MITEs; 2, 0.2\%), centromere-related sequences (19, 1.5\%), ribosomal RNA genes (33, 2.6\%), and other unclassified repeat sequences $(118,9.3 \%)$. In total, such DNA was included in 172 BESs (13.4\% of those harboring repetitive sequences) (Table 2 ).

\section{Functional annotation}

To identify protein-encoding regions, comparison of RepeatMasked BESs with the NCBI non-redundant protein databases revealed that 641 sequences (11.6\%) contained apparent protein-encoding DNA (Table 1). Of these, 252 (39.3\%) showed top BLAST matches search homologies to proteins from $V$. vinifera, whereas 106 (16.5\%) best-matched proteins of O. sativa (Figure 2). This finding is consistent with BLAST data on orchid floral bud ESTs, which yielded top matches to $V$. vinifera followed by O. sativa [12,13]. At first glance, it seems very odd that orchid genes appear to be more highly related to a phylogenetically distant dicot species than to another monocot. Accumulation of additional orchid sequence data is needed to clarify this point.

BLASTN was used to compare the 641 BESs containing protein-encoding sequences to the sequences contained in our orchid EST databases $[12,13]$. We found that 417 BESs (65.1\%) yielded matches and are known to be expressed in orchids (data not shown), whereas 224 (34.9\%) did not show sequence matches when compared with the orchid EST databases.

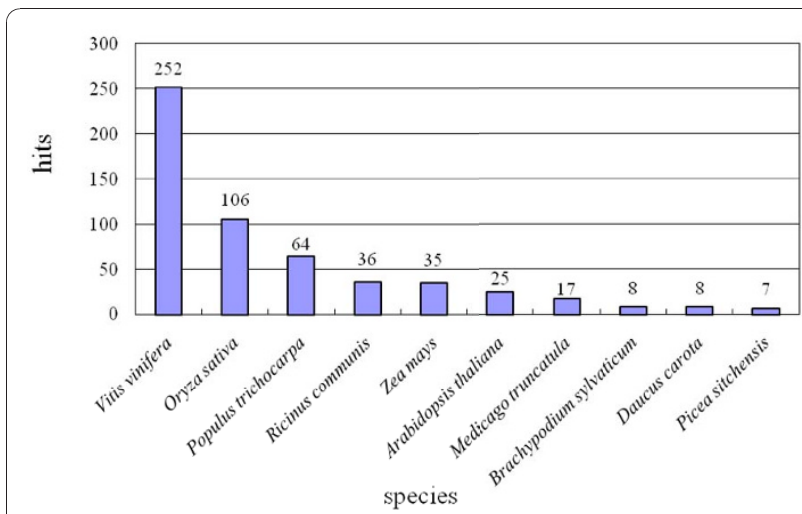

Figure 2 Number of $P$. equestris BAC end sequences (BESs) that had significant hits in the NCBI database. Among the protein matches, the top BLASTX match was to a protein of $V$. vinifera.

Based on the fact that 641 predicted protein-encoding sequences covered $4,544 \mathrm{~kb}$ of the Phalaenopsis genome, as identified from 5,535 BESs, gene density analysis predicted that a gene should occur in every $7.1 \mathrm{~kb}$ of the Phalaenopsis genome. By comparison, banana (M. acuminata) has a gene density of $6.4 \mathrm{~kb}[24]$, rice (O. sativa) is predicted to have a gene every $6.2 \mathrm{~kb}$ [31], whereas $A$. thaliana is thought to have a gene every 4.5 $\mathrm{kb}$ [32].

The 641 BES-derived sequences showing homology to proteins in the NCBI non-redundant protein database were subjected to Gene Ontology (GO) annotation, and divided into three categories: cellular components (321 BESs), molecular functions (182 BESs), and biological processes (329 BESs). Among the 321 BESs in the cellular components category, 30 (9.35\%) corresponded to chloroplast proteins, $25(7.79 \%)$ to membrane proteins, and $18(5.61 \%)$ to other cellular components. However, more than half of these BESs $(179,55.76 \%)$ encoded unknown cellular component proteins (Figure 3a). Sequences in the molecular functions category were distributed as follows: $16.48 \%$ (30 BESs) of unknown molecular function, $15.38 \%$ (28) with transferase activities, $14.84 \%$ (27) with other enzymatic activities, and $14.29 \%$ (26) with involvement in nucleotide binding (Figure $3 \mathrm{~b}$ ). Among the 329 BESs in the biological processes category, more than half corresponded to proteins involved in unknown biological processes (179 BESs, 54.41\%), whereas the rest were associated with other cellular processes $(37,11.25 \%)$, protein metabolism $(17,5.17 \%)$, or transport functions $(17,5.17 \%)$ (Figure 3c).

BLASTX (E-values < 1e-5) was used to compare the RepeatMasked BESs to the protein databases of O. sativa (downloaded from the Rice Annotation Project Database, http://rapdb.dna.affrc.go.jp/download/index. html) and $V$. vinifera (downloaded from the NCBI $V$. vinifera protein database, ftp://ftp.ncbi.nih.gov/ 


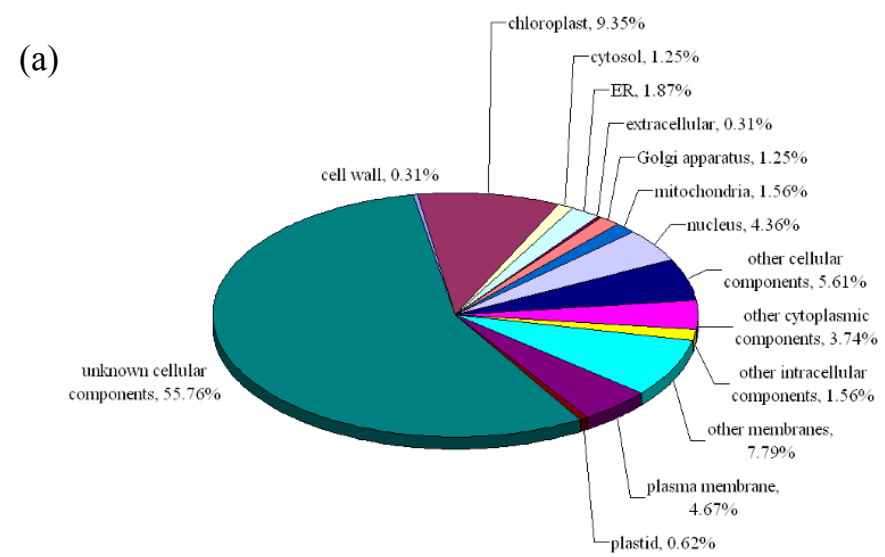

(b)

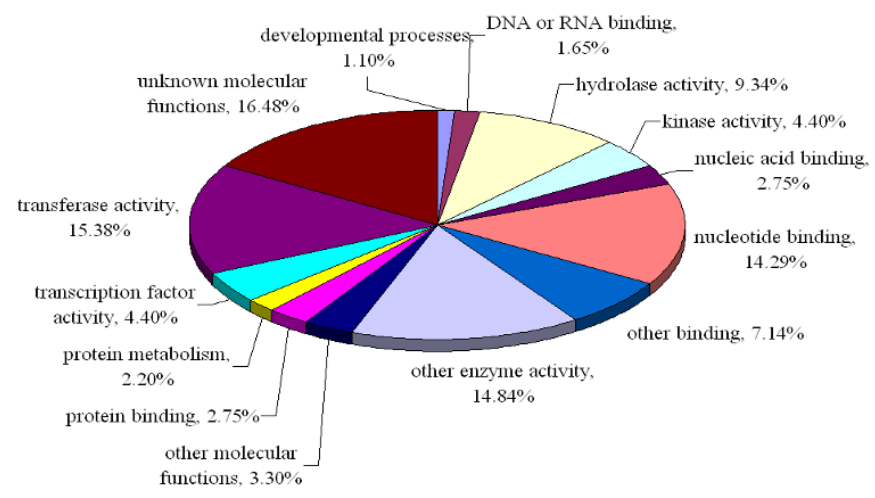

(c)

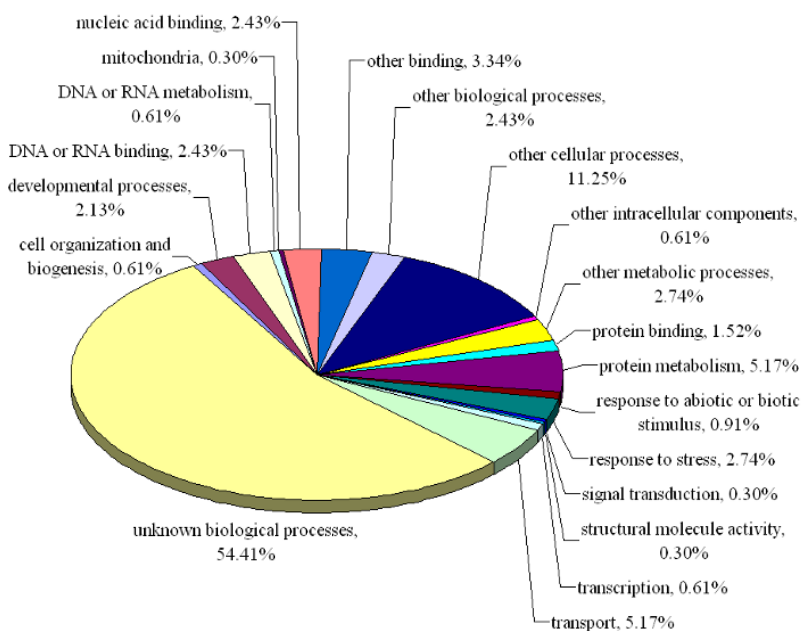

Figure 3 Gene Ontology (GO) analysis of the $P$. equestris BESs into the categories of (a) cellular components, (b) molecular functions, and (c) biological processes. Among the $641 \mathrm{BESs}$ containing protein-encoding regions, 321 were annotated to the cellular components category, 182 to the molecular functions category, and 329 to the biological processes category.

genomes/Vitis_vinifera/protein/). Of the 5,506 BESs, 550 (9.99\%) were homologous to $V$. vinifera proteins. Thus, based on an estimated genome size of 1,600 Mb for $P$. equestris, it may be predicted that the total coding sequences of the $P$. equestris genome might represent approximately $159.8 \mathrm{Mb}$. If an average gene length of $3.4 \mathrm{~kb}$, as in $V$. vinifera [33], is assumed, an estimate of the total gene content of the $P$. equestris genome is 47,007 . When the rice genome was used for comparison, 504 Phalaenopsis BESs showed matches to the rice 
protein database, accounting for $9.15 \%$ of rice proteins. Similar estimations indicate that protein-encoding sequences cover $146.5 \mathrm{Mb}$ of the Phalaenopsis genome and, assuming an average gene length of $2.7 \mathrm{~kb}$ in Oryza [34], predict that the Phalaenopsis genome contains 54,259 genes. These values are comparable to the 30,434 protein-encoding genes identified in the $487-\mathrm{Mb}$ grape genome [33] and the 37,544 protein-encoding genes found in the $389-\mathrm{Mb}$ rice genome [34]. Notably, gene distribution is fairly homogeneous along the chromosomes of rice and Arabidopsis, but genes are distributed more heterogeneously in $V$. vinifera. Pachytene karyotyping analyses of the $P$. equestris genome showed that the distribution of heterochromatin was pericentromeric, suggesting that genes of the Phalaenopsis orchids are more homogeneously distributed (personal communication, Dr. S. B. Chang, Department of Life Sciences, National Cheng Kung University, Taiwan). Based on the genome size of Phalaenopsis, we believe that both average gene length and gene distribution may be similar to those of the rice genome, and that approximately 54,259 heterogeneously distributed genes may be present.

\section{Simple sequence repeats (SSRs)}

We identified 950 SSRs or microsatellites accounting for $17.2 \%$ of the obtained BESs (Table 1), and containing various repeat types (Table 3). BESs from Arabidopsis thaliana, Brassica napus, M. acuminata, O. sativa, $V$. vinifera, and Zea mays were downloaded and analyzed in parallel with those of $P$. equestris. Dinucleotide repeats, which are the most abundant repeat type in $M$. acuminata (47.7\%), B. napus (36.2\%), and $V$. vinifera (28.0\%), were also the most common in the $P$. equestris genome, accounting for $34.37 \%$ of all SSRs. The next most common repeat type in $P$. equestris was mononucleotide in nature (29.9\%) (Table 4$)$. In addition, pentaand tri-nucleotide repeats accounted for $13.5 \%$ and $11.0 \%$, respectively, of all SSRs in the $P$. equestris genome (Table 4). Among the mononucleotide repeats, far more A/T repeats (264 SSRs, 93\%) than $\mathrm{G} / \mathrm{C}$ repeats (20, 7\%) (Table 3) were evident. Among the dinucleotide repeats, AT/TA was the most abundant (253, 77.6\%), followed by AG/CT (47, 14.4\%), AC/GT (24, 7.4\%), and CG/GC (2, 0.6\%) (Table 3$)$. The average distance between SSRs was estimated to be $4.8 \mathrm{~kb}$. Interestingly, this is the highest SSR frequency seen among plant genomes analyzed to date, including those of Arabidopsis, rapeseed (B. napus), banana ( $M$. acuminata), rice, grape, and maize, which are estimated to have an average of $6.4,9.2,6.2,9,5.8$, and $16.1 \mathrm{~kb}$, respectively, between SSRs (Table 4). In $P$. equestris, grape, B. napus, and banana, dinucleotides were found to be the most abundant motifs, whereas trinucleotide SSRs predominated in rice and maize. A. thaliana was particularly rich in
Table 3 Distribution of simple sequence repeats in P. equestris bacterial artificial chromosome (BAC) end sequences

\begin{tabular}{|c|c|c|c|}
\hline Type & No. & Type & No. \\
\hline $\bar{A} / T$ & 264 & AACTC/AGTTG & 1 \\
\hline$C / G$ & 20 & AACTT/AATTG & 1 \\
\hline $\mathrm{AC} / \mathrm{GT}$ & 24 & AAGAG/CTCTT & 3 \\
\hline $\mathrm{AG} / \mathrm{CT}$ & 47 & AAGGG/CCCTT & 1 \\
\hline AT/AT & 253 & AAGTT/AATTC & 1 \\
\hline $\mathrm{CG} / \mathrm{CG}$ & 2 & AAGCC/CGGTT & 1 \\
\hline $\mathrm{AAC} / \mathrm{GTT}$ & 22 & AATAT/ATATT & 4 \\
\hline $\mathrm{AAG} / \mathrm{CT}$ & 12 & AATCC/AGGTT & 1 \\
\hline AAT/ATT & 51 & AATGC/ACGTT & 1 \\
\hline ACC/GGT & 3 & ACACG/CTGTG & 1 \\
\hline ACT/ATG & 2 & ACCTC/AGTGG & 1 \\
\hline AGC/CGT & 1 & ACGAG/CTCTG & 1 \\
\hline $\mathrm{AGG} / \mathrm{CCT}$ & 6 & ACTAT/ATATG & 1 \\
\hline AGT/ATC & 5 & AGAGG/CCTCT & 4 \\
\hline $\mathrm{CCG} / \mathrm{CGG}$ & 2 & AGGAT/ATCCT & 1 \\
\hline AAAG/CTT & 2 & AGGGC/CCCGT & 1 \\
\hline AAAT/ATTT & 20 & AGGGG/CCCCT & 1 \\
\hline AACT/ATTG & 1 & AAAAAG/CTTITT & 11 \\
\hline AАTT/AATT & 4 & AAAAAT/ATTTTT & 10 \\
\hline ACAG/CTGT & 1 & AAAACC/GGTITT & 15 \\
\hline ACAT/ATGT & 12 & AAAATT/AATTTT & 1 \\
\hline ACCT/ATGG & 4 & AAAATC/AGTTT & 2 \\
\hline ACTC/AGTG & 1 & AAACCT/ATTTGG & 1 \\
\hline AGAT/ATCT & 2 & AAACCC/GGGTTT & 1 \\
\hline AGGG/CCCT & 2 & AAAGTC/AGTTTC & 1 \\
\hline ACGT/ATGC & 1 & AAGGGC/CCCGTT & 1 \\
\hline AAAAC/GTITT & 10 & AACGTG/ACTTGC & 4 \\
\hline AAAAG/CTITT & 20 & AAGATC/AGTTCT & 1 \\
\hline AAAAT/ATTTT & 34 & AATATT/AATTAT & 1 \\
\hline AAACT/ATTTG & 2 & ACATAG/ATCTGT & 1 \\
\hline AAAGT/ATTTC & 2 & ACATAT/ATATGT & 2 \\
\hline AAAGG/CCTIT & 1 & ACCTGC/ACGTGG & 2 \\
\hline AAATC/AGTTT & 2 & AACCCT/ATTGGG & 1 \\
\hline AAATG/ACTIT & 1 & ACACCC/GGGTGT & 1 \\
\hline AAATT/AATTT & 30 & ACCCCT/ATGGGG & 1 \\
\hline AACAC/GTGTT & 1 & AGAGGC/CCGTCT & 1 \\
\hline
\end{tabular}

mononucleotide repeats. We noted some among-study variations in reported frequencies [24,26]; these appear to be mainly attributable to the use of different criteria for identifying SSRs. However, we can generally conclude that $P$. equestris and dicot plants such as grapevine, $B$. napus, and $A$. thaliana contain a high proportion of AT-rich motifs. The greatest repeat number (275) identified to date is found in the orchid genome (this study), whereas other monocots, such as O. sativa and $Z$. mays, appear to contain lower proportions of AT-rich motifs (Figure 4). 
Table 4 Distribution and frequency of simple sequence repeats (SSRs) detected in different plant species

\begin{tabular}{|c|c|c|c|c|c|c|c|}
\hline & A. thaliana & B. napus & M. acuminata & O. sativa & P. equestris & V. vinifera & Z. mays \\
\hline No. of BESs & 26,068 & 88,825 & 6,376 & 78,427 & 5,506 & 31,907 & 54,960 \\
\hline Total sequence length (bp) & $13,987,589$ & $39,551,595$ & $4,517,901$ & $69,423,321$ & $4,520,220$ & $18,117,956$ & $37,410,959$ \\
\hline Mononucleotides & $40.3(878)$ & $7.5(320)$ & $0.8(6)$ & $9.1(696)$ & $29.9^{\mathrm{a}}(284)^{\mathrm{b}}$ & $6.0(188)$ & $7.2(167)$ \\
\hline Dinucleotides & $13.1(285)$ & $36.2(1,551)$ & $47.7(350)$ & $19.9(1,531)$ & $34.3(326)$ & $28.0(881)$ & $15.4(358)$ \\
\hline Trinucleotides & $21.2(462)$ & $20.4(876)$ & $20.6(151)$ & $28.9(2,219)$ & $11.0(104)$ & $18.7(586)$ & $35.7(831)$ \\
\hline Tetranucleotides & $3.9(84)$ & $6.9(294)$ & $9.0(66)$ & $10.2(783)$ & $5.3(50)$ & $14.9(467)$ & $8.3(193)$ \\
\hline Pentanucleotides & $15.3(333)$ & $21.2(911)$ & $13.1(96)$ & $21.4(1,642)$ & $13.5(128)$ & $21.4(673)$ & $21.6(504)$ \\
\hline Hexanucleotides & $6.3(137)$ & 7.9 (338) & $8.9(65)$ & $10.5(804)$ & $6.1(58)$ & $11.0(347)$ & $11.9(276)$ \\
\hline Total SSRs & 2,179 & 4,290 & 734 & 7,675 & 950 & 3,142 & 2,329 \\
\hline SSR frequency ${ }^{c}$ & 6.4 & 9.2 & 6.2 & 9 & 4.8 & 5.8 & 16.1 \\
\hline Most frequent SSR motif & $\mathrm{A} / \mathrm{T}$ & AT/TA & AT/TA & $\mathrm{CCG} / \mathrm{CGG}$ & $\mathrm{A} / \mathrm{T}$ & AT/TA & $\mathrm{AGC} / \mathrm{GCT}$ \\
\hline
\end{tabular}

${ }^{\text {a }}$ Percentage of SSRs in each category.

${ }^{b}$ Number of SSRs in each category.

c Average estimated distance between SSRs (kb).

SSR markers have been widely used for genotyping of crop plant species $[35,36]$. Of the 950 detected SSRs, we chose 206 for use in primer design (Additional file 1), and subsequently assessed whether the primers could successfully distinguish 12 Phalaenopsis species, based on allelic polymorphisms. More than $85 \%$ of primer pairs successfully amplified products from at least 1 of the 12 tested Phalaenopsis species, all of which have been extensively used as parents in breeding programs (Additional file 2). The cross-species transferability rate of the tested SSRs ranged from 76.1-54.8\% (Additional file 2), and most primer pairs produced polymorphic bands in the majority of tested Phalaenopsis species (Additional file 3). In a future study, we will examine the efficacy of such SSR markers for genotyping of commercial orchid cultivars.

\section{Comparative mapping of orchid BAC ends to other plant genomes for identification of microsynteny}

To examine syntenic relationships between orchid and other plant species, orchid BESs were BLAST-searched against the whole-genome sequences of $A$. thaliana, rice (O. sativa), poplar (Populus trichocarpa), and grape ( $V$. vinifera). BAC end pairs of appropriate orientation and no more than 50-300 apart on any given chromosome were considered to be potentially collinear with the target genome. Our results revealed that $142 \mathrm{Pha}$ laenopsis BESs, including 14 end-pair sequences, yielded

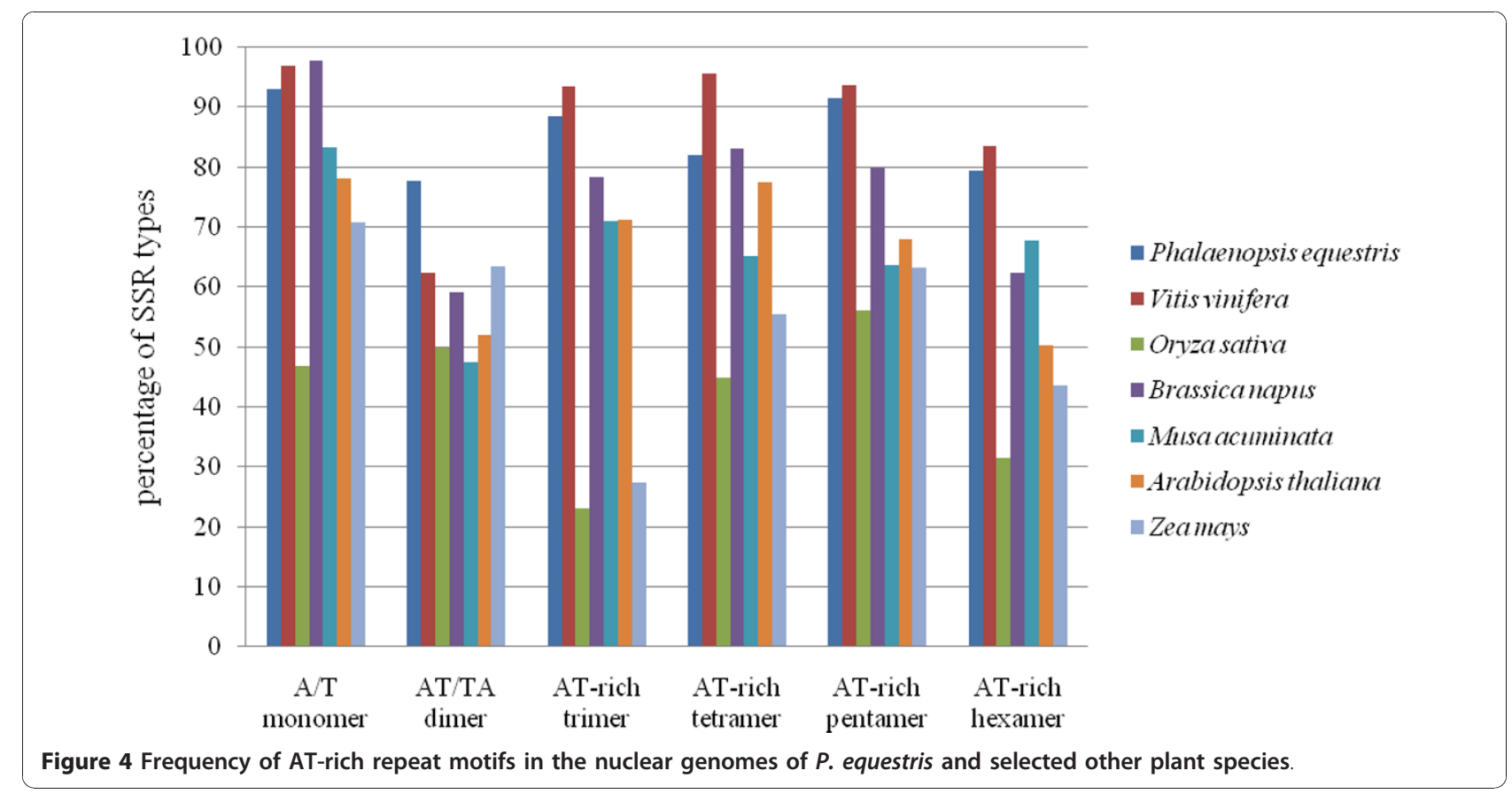


hits in the poplar genome. Twelve pairings mapped together on various chromosomes, but only 1 such pair was found within 50-300 kb of another, suggesting colinearity (Table 5). The next greatest number of hits was obtained when the grape genome was compared with that of our BESs; significant hits were obtained for 123 $\mathrm{BESs}$, including $12 \mathrm{BAC}$ end-pairs, 10 of which mapped together on various chromosomes. One of the $10 \mathrm{BAC}$ end-pairs was found on different contigs of the same chromosome; presumably these contigs are mutually close, maybe even within 50-300 kb (Table 5). Ninetyfour orchid BESs, including 12 paired ends, showed significant hits to the Arabidopsis genome. Eleven of the BAC end-pairs colocalized on various chromosomes, but none were less than 50-300 kb apart (Table 5). The mapping of orchid BESs to the genome of rice produced 83 BES hits, including six paired ends that colocalized on various chromosomes, but were not within 50-300 $\mathrm{kb}$ of each other (Table 5).

The simple Monte Carlo Test [37] was used to assess the statistical significance of the microsynteny results. The sequences of each Phalaenopsis BES were randomly shuffled 100 times to obtain 550,600 simulated sequences, which were next BLASTN- compared to the genomic sequences of poplar, grape, rice, and Arabidopsis. None of the simulated sequences mapped to the genomes of the various plants, suggesting that our results with respect to microsynteny mapping of orchid BESs onto other plant genomes are meaningful.

Most paired ends that mapped together on plant chromosomes were annotated as ribosomal DNA (rDNA); these sequences accounted for 10 of 14 end-pairs in poplar, 10 of 12 in grape and Arabidopsis, and all 6 end-pairs of rice. In addition, all end-pairs that contained rDNA mapped to a single chromosome in each plant species.

Twenty-nine orchid BESs containing Phalaenopsis chloroplast genome sequences showed matches with genomic sequences of the four plant genomes: $25 \mathrm{BESs}$ with the grape genome, 24 with the rice genome, 21 with the poplar genome, and 2 with the Arabidopsis

Table 5 Microsynteny between Phalaenopsis and A. thaliana, O. sativa, P. trichocarpa and V. vinifera

\begin{tabular}{lcccc}
\hline & $\begin{array}{c}\text { A. } \\
\text { thaliana }\end{array}$ & $\begin{array}{c}\text { O. } \\
\text { sativa }\end{array}$ & $\begin{array}{c}\boldsymbol{P} \text {. } \\
\text { trichocarpa }\end{array}$ & $\begin{array}{c}\boldsymbol{V} \text {. } \\
\text { vinifera }\end{array}$ \\
\hline No. of hits & 94 & 83 & 142 & 123 \\
Pair ends & 12 & 6 & 14 & 12 \\
Same chromosome & 11 & 6 & 12 & 10 \\
50- to 300-kb & 0 & 0 & 1 & $1^{\text {a }}$ \\
sequence & & & &
\end{tabular}

a The whole-genome sequence data of $V$. vinifera shows that each individual chromosome contains several contigs. Thus, we were unable to determine the exact distances between the BAC pair ends that mapped together on the various chromosomes of the grape genome. genome (Table 6; assessed using BLASTN with an E-value <1e-30). Moreover, such BESs also showed matches within chloroplast DNA sequences from the four plant genomes: 28 with the grape genome, 24 with rice, 27 with poplar, and 25 with Arabidopsis (E-value $<1 \mathrm{e}-30)$. Transfer of chloroplast DNA to the nucleus is well-known to result in insertion of chloroplast DNA into nuclear chromosomes. In rice, 421-453 chloroplast insertions have been identified throughout the 12 chromosomes, forming $0.18-0.19 \%$ of the rice nuclear genome [34]. In the present work, the 29 orchid BESs containing Phalaenopsis chloroplast DNA included 12 paired and 5 unpaired BES end sequences. This suggests that at least some of these BESs may be located in the Phalaenopsis nuclear genome, rather than representing experimental contamination with chloroplast DNA.

Previous reports found negligible colinearity between onion (Asparagales) and rice (Poales) $[38,39]$. The Asparagales include a number of economically important plants, such as asparagus, chives, garlic, leeks, onions, and orchids. Similarly, the well-documented high-level synteny among grass genomes is not found among members of other monocot orders [e.g., Musa (Zingiberales)], even though microsynteny persisted beyond the time of divergence of the Commelinid orders Poales and Zingiberales [40]. In the present work, we failed to find any syntenic relationship between orchid and rice sequences, confirming the previously noted lack of synteny between Asparagales and Poales.

Whole-genome duplication, resulting in polyploidy, occurred in early monocots such as the Poales and Zingiberales $[40,41]$. Duplication, and gene loss and rearrangements occurring after such whole-genome duplication, led to subsequent evolution and increases in morphological complexity. This may also have occurred in the orchid genome, as suggested by the presence of the four AP3-like paralogs that form the basis for the complicated floral morphologies of Phalaenopsis [16]. Interestingly, these paralogs are present in at least four out of the five subfamilies of the Orchidaceae [42]. Future wholegenome sequencing of Phalaenopsis should provide additional insights into genome reorganization and help to clarify differences in genomic composition between orchids and other plant species.

Table 6 Number of bacterial artificial chromosome (BAC) end sequences (BESs) containing Phalaenopsis chloroplast DNA with hits to the nuclear and chloroplast DNA of A. thaliana, O. sativa, P. trichocarpa and V. vinifera

\begin{tabular}{lcccc}
\hline & A. thaliana & O. sativa & P. trichocarpa & V. vinifera \\
\hline Nuclear DNA & 2 & 24 & 21 & 25 \\
Chloroplast DNA & 25 & 24 & 27 & 28
\end{tabular}




\section{Conclusions}

This analysis of Phalaenopsis BAC end sequences offers the first insights into the composition of the Phalaenopsis genome in terms of GC content, transposable elements present, protein-encoding regions, SSRs, and potential microsynteny between Phalaenopsis and other plant species. The protein sequence similarities between Phalaenopsis and grape and the potential microsynteny between Phalaenopsis and poplar are interesting and should be confirmed by large-scale BAC end sequencing. The present work also provides a good basis for additional sequence analysis of Phalaenopsis BAC libraries, and will also encourage contig fingerprinting and physical mapping of the Phalaenopsis genome.

\section{Methods}

\section{Orchid BAC-end sequencing}

BAC clones were randomly chosen from 96-well microplates and inoculated into 96-well deep-well plates containing $1.5 \mathrm{ml}$ of $2 \mathrm{x} \mathrm{LB}$ medium with $12.5 \mu \mathrm{g} / \mathrm{ml}$ chloramphenicol. Plates were incubated at $37^{\circ} \mathrm{C}$ with continuous shaking at $100 \mathrm{rpm}$ for 20-24 h. BAC-end sequencing was performed using BigDye ${ }^{\mathbb{B}}$ Terminator $\mathrm{v}$ 1.1 and ABI PRISM ${ }^{\circledR} 3730$ DNA Analyzer technologies (Applied Biosystems, Life Technologies Corporation, Foster City, CA). The work was performed by the Sequencing Core Facility of the National Yang Ming University Genome Center (YMGC, Taipei, Taiwan) and the Arizona Genomics Institute DNA Sequencing Center (AGI, Tucson, AZ).

BESs were base-called and processed using CodonCode Aligner software (Version 2.0; CodonCode Corporation, Dedham, MA), which integrates the PHRED program [43]. We used the default parameters of "maximize region with error rate $<0.1$ " to trim bases from flanking sequences and next performed the operations "move all sequences shorter than 25 bases to trash" and "move all sequences with fewer than 50 Phred-20 bases to trash." Vector sequences were trimmed using Sequencher V4.1 (Gene Codes Corporation, Ann Arbor, $\mathrm{MI}$ ) with reference to the pIndigoBAC5 DNA sequence. We next discarded all BESs $<100 \mathrm{bp}$, which yielded a set of 5,535 BESs. These were BLASTN-searched against the chloroplast DNA sequence of $P$. aphrodite subsp. formosana (GenBank accession no. NC_007499) [44] and the mitochondrial DNA sequence of $O$. sativa (japonica) cultivar Nipponbare (accession no. DQ167400) [45], using a stringent threshold of $<1$ e-50.

\section{Analysis of repetitive sequences}

BESs were analyzed for repetitive sequences using RepeatMasker [46]. We applied the same default conditions as were employed in construction of the Arabidopsis, rice, and maize sections of the RepBase Update databases [47]. We next used BLASTN and TBLASTX to search the TIGR plant repeat database (downloaded on Sep. 5, 2009) [48] with E-values $<1 \mathrm{e}-10$ and $<1 \mathrm{e}-5$, respectively. Repetitive sequences were annotated using the RepeatMasker default setting or were classified employing the TIGR codes for repetitive plant sequences.

\section{Functional annotation of Phalaenopsis BESs}

The 5,506 RepeatMasked BESs were further analyzed for protein-encoding regions via BLASTX searching of NCBI non-redundant protein databases (E-values $<1 \mathrm{e}-5)$. Protein-encoding BESs were BLASTN-searched against our in-house orchid EST databases [12,13], using an E-value < 1e-20. BESs containing proteinencoding regions homologous to proteins found in the NCBI non-redundant protein databases were further analyzed in terms of Gene Ontology (GO) annotations, using a BLASTX search of the Arabidopsis genome annotation database (ftp://ftp.arabidopsis.org/home/ tair/Genes/TAIR9_genome_release/; E-value < 1e-7). Categories were assigned based on biological, functional, and molecular annotations available from GO http://www.geneontology.org/.

\section{SSR identification and marker development}

BESs from A. thaliana, B. napus, M. acuminata, O. sativa, $V$. vinifera, and $Z$. mays were obtained from the Genome Survey Sequences (GSS) database of the NCBI (downloaded on Dec. 11, 2009), and subjected to SSR analysis using the same parameters as were employed in our analysis of orchid BESs. SSR types (mononucleotide to hexanucleotide) were identified using the MIcroSAtellite (MISA) tool [49]; the analysis required a minimum length of 20 bases for mononucleotide repeats and at least 15 bases for dinucleotide-to-hexanucleotide repeats, and allowed a maximum of an 100-nt interruption if compound repeats were encountered. Primer3 software http://frodo.wi.mit.edu/primer3/ was used for primer design. The following parameters were employed: (1) BESs with a minimum of eight dinucleotide, five trinucleotide, four tetranucleotide, three pentanucleotide, or three hexanucleotide repeats; or an SSR motif length longer than $15 \mathrm{bp}$; (2) primer lengths of 18-25 nt, with 20 nt being considered optimal; and (3) predicted PCR products of $150-350 \mathrm{bp}$. A total of 206 primer pairs (Additional file 1) were synthesized and used to amplify genomic DNA from 12 Phalaenopsis species: $P$. amabilis, $P$. aphrodite subsp. formosana, P. schilleriana, $P$. stuartiana, $P$. equestris, $P$. sanderiana, P. lueddemanniana, P. amboinensis, P. pulcherrima, P. fasciata, P. venosa, and P. gigantea. Genomic DNA was isolated from leaf samples using a BioKit Plant Genomic DNA Purification Kit. PCR was performed 
using 10 ng of genomic DNA, paired primers, dNTPs, $10 \times$ buffer, and Taq polymerase, in $20-\mu l$ reaction volumes. The PCR amplification conditions were: $94^{\circ} \mathrm{C}$ for $5 \mathrm{~min}$ followed by 45 cycles of $94^{\circ} \mathrm{C}$ for $60 \mathrm{sec}$, annealing $\left(45-60^{\circ} \mathrm{C}\right)$ for $40 \mathrm{sec}$ and $72^{\circ} \mathrm{C}$ for $40 \mathrm{sec}$, and a final extension for $5 \mathrm{~min}$ at $72^{\circ} \mathrm{C}$. PCR products were separated on either $3 \%(\mathrm{w} / \mathrm{v})$ agarose or $8 \%(\mathrm{w} / \mathrm{v})$ denaturing polyacrylamide gels, which were next stained with ethidium bromide for visualization of SSR bands.

\section{Microsynteny between $P$. equestris and $A$. thaliana,} $O$. sativa, $P$. trichocarpa, and $V$. vinifera

Phalaenopsis BESs (not RepeatMasked) were compared with the genomic sequences of $A$. thaliana, O. sativa, $P$. trichocarpa, and $V$. vinifera (downloaded from the NCBI database ftp://ftp.ncbi.nih.gov/genomes/ on Feb. $27,2010)$ by means of a BLASTN search with an E-value $<1 \mathrm{e}-10$. To identify BACs from the Phalaenopsis library that showed microsynteny with the reference genomes, as described in a previous study on Musa [24], we searched the Phalaenopsis genomic sequence for BESs, both ends of which showed highly significant matches to $A$. thaliana, O. sativa, $P$. trichocarpa, or $V$. vinifera sequences, and that were located 50-300 kb apart in the Phalaenopsis genome.

The simple Monte Carlo Test [37] was used to assess the statistical significance of microsynteny between Phalaenopsis BESs and the genomes of $A$. thaliana, $O$. sativa, $P$. trichocarpa, and $V$. vinifera. The sequence of each Phalaenopsis BES was randomly shuffled 100 times to obtain 550,600 simulated sequences, which were then compared, using BLASTN, to the Arabidopsis, rice, poplar, and grape genomes (E-value $<1 \mathrm{e}-10)$.

\section{Additional material}

Additional file 1: List of 206 SSR markers developed from the $P$. equestris BAC end sequences. The data provided represent details of the SSR markers e.g. marker name, accession number, primer sequence, PCR condition, expected product size and indication of whether prime pairs successfully amplified at least one of the 12 tested Phalaenopsis species.

Additional file 2: Rate of successful amplification of $P$. equestris SSRs among 12 Phalaenopsis species. The cross-species amplification rate of the 206 SSR markers was assessed in 12 Phalaenopsis species.

Additional file 3: PCR amplification profiles of four $P$. equestris SSR markers in 12 Phalaenopsis species. 12 Phalaenopsis species listed in Additional file 2 were used for polymorphism analysis of four SSR markers PeGBMS114, PeGBMS117, PeGBMS126 and PeGBMS216 listed in Additional file 1

\section{List of abbreviations}

BAC: bacterial artificial chromosome; BESs: BAC end sequences; GO: Gene Ontology; MISA: MIcroSAtellite identification tool; SSRs: simple sequence repeats;

\section{Acknowledgements}

We thank Dr. Michel Delseny (Laboratory of Plant Genome and Plant Physiology, University of Perpignan, France) for helpful discussions and critical reading of the manuscript. We acknowledge the technical services provided by the Sequencing Core Facility at the National Yang-Ming University Genome Research Center (YMGC, Taipei, Taiwan). The Sequencing Core Facility is supported by the National Research Program for Genomic Medicine (NRPGM) of the National Science Council, Taiwan. We also acknowledge the technical services provided by the Arizona Genomics Institute (AGI) DNA Sequencing Center and the AGI Physical Mapping Center, Arizona, USA. This work was supported by grants 98-2321-B-006-004MY3 from National Science Council, Taiwan, and 98AS-1.2.1-ST-a4 and 100AS-1.2.2-ST-a2 from Council of Agriculture, Taiwan.

\section{Author details}

'Department of Life Sciences, National Cheng Kung University, Tainan 701, Taiwan. ${ }^{2}$ Institute of Tropical Plant Sciences, National Cheng Kung University, Tainan 701, Taiwan. ${ }^{3}$ Orchid Research Center, National Cheng Kung University, Tainan 701, Taiwan

\section{Authors' contributions}

$\mathrm{CCH}$ performed the bioinformatic analyses for genes, transposable elements, and microsynteny. YLC, YLL, and YTK performed the SSR prediction and verification. TCC and WLW constructed the Phalaenopsis BAC library for sequencing. WCT, YYH, YWC, WLW and HHC participated in the study design and data analysis. CCH, YLC, YLL, WLW, and HHC drafted the manuscript. All authors read and approved the final manuscript.

Received: 19 July 2010 Accepted: 6 January 2011

Published: 6 January 2011

\section{References}

1. Atwood JT: The size of Orchidaceae and the systematic distribution of epiphytic orchids. Selbyana 1986, 9:16.

2. Cozzolino S, Widmer A: Orchid diversity: an evolutionary consequence of deception? Trends Ecol Evol 2005, 20:487-494.

3. Tremblay RL, Ackerman JD, Zimmerman JK, Calvo RN: Variation in sexual reproduction in orchids and its evolutionary consequences: a spasmodic journey to diversification. Biol J Linn Soc 2005, 84:1-54.

4. Otero JT, Flanagan NS: Orchid diversity-beyond deception. Trends Ecol Evol 2006, 21:64-65.

5. Gravendeel B, Smithson A, Slik FJ, Schuiteman A: Epiphytism and pollinator specialization: drivers for orchid diversity? Philos Trans $R$ SoC Lond B Biol Sci 2004, 359:1523-1535.

6. Crane PR, Friis EM, Pedersen KR: The origin and early diversification of angiosperm. Nature 1995, 374:27-33.

7. Dressler RL: Phylogeny and Classification of the Orchid Family Portland: Dioscorides Press; 1993

8. Ramirez SR, Gravendeel B, Singer RB, Marshall CR, Pierce NE: Dating the origin of the Orchidaceae from a fossil orchid with its pollinator. Nature 2007, 448:1042-1045.

9. $\mathrm{Yu} \mathrm{H}$, Goh CJ: Molecular genetics of reproductive biology in orchids. Plant Physiol 2001, 127:1390-1393.

10. Schiestl FP, Peakall R, Mant JG, Ibarra F, Schulz C, Franke S, Francke W: The chemistry of sexual deception in an orchid-wasp pollination system. Science 2003, 302:437-438.

11. Tsai WC, Hsiao YY, Pan ZJ, Kuoh CS, Chen WH, Chen HH: The role of ethylene in orchid ovule development. Plant Sci 2008, 175:98-105.

12. Peakall R: Speciation in the Orchidaceae: confronting the challenges. $\mathrm{Mol}$ Ecol $2007,16 \cdot 2834-2837$.

13. Tsai WC, Hsiao YY, Lee SH, Tung CW, Wang DP, Wang HC, Chen WH, Chen $\mathrm{HH}$ : Expression analysis of the ESTs derived from the flower buds of Phalaenopsis equestris. Plant Sci 2006, 170:426-432.

14. Hsiao YY, Tsai WC, Kuoh CS, Huang TH, Wang HC, Wu TS, Leu YL, Chen WH, Chen $\mathrm{HH}$ : Comparison of transcripts in Phalaenopsis bellina and Phalaenopsis equestris (Orchidaceae) flowers to deduce monoterpene biosynthesis pathway. BMC Plant Biol 2006, 6:14

15. Tan J, Wang HL, Yeh KW: Analysis of organ-specific, expressed genes in Oncidium orchid by subtractive expressed sequence tags library. Biotechnol Lett 2005, 27:1517-1528. 
16. Tsai WC, Kuoh CS, Chuang MH, Chen WH, Chen HH: Four DEF-like MADS box genes displayed distinct floral morphogenetic roles in Phalaenopsis orchid. Plant Cell Physiol 2004, 45:831-844.

17. Tsai WC, Lee PF, Chen HI, Hsiao YY, Wei WJ, Pan ZJ, Chuang MH, Kuoh CS, Chen WH, Chen HH: PeMADS6, a GLOBOSA/PISTILLATA-like gene in Phalaenopsis equestris involved in petaloid formation, and correlated with flower longevity and ovary development. Plant Cell Physiol 2005, 46:1125-1139.

18. Hsiao YY, Jeng MF, Tsai WC, Chuang YC, Li CY, Wu TS, Kuoh CS, Chen WH, Chen $\mathrm{HH}$ : A novel homodimeric geranyl diphosphate synthase from the orchid Phalaenopsis bellina lacking a DD(X)2-4D motif. Plant J 2008, 55:719-733.

19. Wang CY, Chiou CY, Wang HL, Krishnamurthy R, Venkatagiri S, Tan J, Yeh KW: Carbohydrate mobilization and gene regulatory profile in the pseudobulb of Oncidium orchid during the flowering process. Planta 2008, 227:1063-1077.

20. Kao YY, Chang SB, Lin TY, Hsieh $\mathrm{CH}$, Chen YH, Chen WH, Chen CC: Differential accumulation of heterochromatin as a cause for karyotype variation in Phalaenopsis orchids. Ann Bot-London 2001, 87:387-395.

21. Lin S, Lee HC, Chen WH, Chen CC, Kao YY, Fu YM, Chen YH, Lin TY: Nuclear DNA Contents of Phalaenopsis sp. and Doritis pulcherrima. J Amer SoC Hort Sci 2001, 126:195-199.

22. Shultz JL, Kazi S, Bashir R, Afzal JA, Lightfoot DA: The development of BACend sequence-based microsatellite markers and placement in the physical and genetic maps of soybean. Theor Appl Genet 2007, 114:1081-1090.

23. Lai CWJ, Yu QY, Hou S, Skelton RL, Jones MR, Lewis KLT, Murray J, Eustice M, Guan P, Agbayani R, Moore PH, Ming R, Presting GG: Analysis of papaya $B A C$ end sequences reveals first insights into the organization of a fruit tree genome. Mol Genet Genomics 2006, 276:1-12.

24. Cheung F, Town CD: A BAC end view of the Musa acuminata genome. BMC Plant Biology 2007, 7:29.

25. Han Y, Korban SS: An overview of the apple genome through BAC end sequence analysis. Plant Mol Biol 2008, 67:581-588.

26. Cavagnaro PF, Chung SM, Szklarczyk M, Grzebelus D, Senalik D, Atkins AE, Simon PW: Characterization of a deep-coverage carrot (Daucus carota L.) BAC library and initial analysis of BAC-end sequences. Mol Genet Genomics 2009, 281:273-288.

27. Wing RA, Ammiraju JS, Luo M, Kim H, Yu Y, Kudrna D, Goicoechea JL, Wang W, Nelson W, Rao K, Brar D, Mackill DJ, Han B, Soderlund C, Stein L, SanMiguel P, Jackson S: The Oryza map alignment project: the golden path to unlocking the genetic potential of wild rice species. Plant $\mathrm{Mol}$ Biol 2005, 59:53-62.

28. Ammiraju JS, Luo M, Goicoechea JL, Wang W, Kudrna D, Mueller C, Talag J, Kim H, Sisneros NB, Blackmon B, Fang E, Tomkins JB, Brar D, MacKill D, McCouch S, Kurata N, Lambert G, Galbraith DW, Arumuganathan K, Rao K, Walling JG, Gill N, Yu Y, SanMiguel P, Soderlund, Jackson S, Wing RA: The Oryza bacterial artificial chromosome library resource: construction and analysis of 12 deep-coverage large-insert BAC libraries that represent the 10 genome types of the genus Oryza. Genome Res 2006, 16:140-147.

29. Capesius I, Nagl W: Molecular and cytological characteristics of nuclear DNA and chromatin for agniosperm systematics: DNA diversification in the evolution of four orchids. Plant Syst Evol 1978, 129:143-166.

30. Terol J, Naranjo MA, Ollitrault P, Talon M: Development of genomic resources for Citrus clementina: characterization of three deep-coverage BAC libraries and analysis of 46,000 BAC end sequences. BMC Genomics 2008, 9:423.

31. Yuan Q, Ouyang S, Wang A, Zhu W, Maiti R, Lin H, Hamilton J, Haas B, Sultana R, Cheung F, Wortman J, Buell CR: The institute for genomic research Osa1 rice genome annotation database. Plant Physiol 2005 138:18-26

32. The Arabidopsis Genome Initiative: Analysis of the genome sequence of the flowering plant Arabidopsis thaliana. Nature 2000, 408:796-815.

33. Jaillon O, Aury JM, Noel B, Policriti A, Clepet C, Casagrande A, Choisne N, Aubourg S, Vitulo N, Jubin C, Vezzi A, Legeai F, Hugueney P, Dasilva C, Horner D, Mica E, Jublot D, Poulain J, Bruye're C, Billault A, Segurens B, Gouyvenoux M, Ugarte E, Cattonaro F, Anthouard F, Vico V, Fabbro CD, Alaux M, Gaspero GD, Dumas $V$, et al: The grapevine genome sequence suggests ancestral hexaploidization in major angiosperm phyla. Nature 2007, 449:463-467.
34. International Rice Genome Sequencing Project: The map-based sequence of the rice genome. Nature 2005, 436:793-800.

35. Hayden MJ, Nguyen TM, Waterman A, McMichael GL, Chalmers KJ: Application of multiplex-ready PCR for fluorescence-based SSR genotyping in barley and wheat. Mol Breed 2008, 21:271-281.

36. Singh H, Deshmukh RK, Singh A, Singh AK, Gaikwad K, Sharma TR, Mohapatra T, Singh NK: Highly variable SSR markers suitable for rice genotyping using agarose gels. Mol Breed 2010, 25:359-364.

37. Hope ACA: A simplified Monte Carlo significance test procedure. J Stat Soc (Ser B) 1968, 30:582-598.

38. Martin WJ, McCallum J, Shigyo M, Jakse J, Kuhl JC, Yamane N, Joyce MP, Gokce AF, Sink KC, Town CD, Havey MJ: Genetic mapping of expressed sequences in onion and in silico comparisons with rice show scant colinearity. Mol Gen Genomics 2005, 274:197-204.

39. Jakse J, Telgmann A, Jung C, Khar A, Melgar S, Cheung F, Town CD, Havey MJ: Comparative sequence and genetic analyses of asparagus BACs reveal no microsynteny with onion or rice. Theor Appl Genet 2006 114:31-39

40. Lescot M, Piffanelli P, Ciampi AY, Ruiz M, Blanc G, Mack JL, da Silva FR, Santos CMR, D'Hont A, Garsmeur O, Vilarinhos AD, Kanamori $H$ Matsumoto T, Ronning CM, Cheung F, Haas BJ, Althoff R, Arbogast T, Hine E, Pappas GJ, Sasaki T, Souza MT, Miller RNG, Glaszmann JC, Town CD: Insights into the Musa genome: Syntenic relationships to rice and between Musa species. 2008, 9:58.

41. Tang H, Bowers JE, Wang X, Paterson AH: Angiosperm genome comparisons reveal early polyploidy in the monocot lineage. Proc Natl Acad Sci USA 2010, 107:472-477.

42. Mondragon-Palomino M, Theissen G: MADS about the evolution of orchid flowers. Trends Plant Sci 2008, 13:51-59.

43. Ewing B, Hillier L, Wendl MC, Green P: Base-calling of automated sequencer traces using phred. I. Accuracy assessment. Genome Res 1998, 8:175-185

44. Chang CC, Lin HC, Lin IP, Chow TY, Chen HH, Chen WH, Cheng CH, Lin CY, Liu SM, Chaw SM: The chloroplast genome of Phalaenopsis aphrodite (Orchidaceae): comparative analysis of evolutionary rate with that of grasses and its phylogenetic implications. Mol Biol Evol 2006, 23:279-291.

45. Tian X, Zheng J, Yu J: The rice mitochondrial genomes and their variations. Plant Physiol 2006, 140:401-410.

46. Smit AFA, Hubley R, Green P: RepeatMasker Open-3.0. 1996 [http://www. repeatmasker.org]

47. Jurka J, Kapitonov W, Pavlicek A, Klonowski P, Kohany O, Walichiewicz J: Repbase Update, a database of eukaryotic repetitive elements. Cytogenet Genome Res 2005, 110:462-467.

48. Ouyang S, Buell CR: The TIGR Plant Repeat Databases: a collective resource for the identification of repetitive sequences in plants. Nucleic Acids Res 2004, 32:360-363.

49. Thiel T, Michalek W, Varshney RK, Graner A: Exploiting EST databases for the development and characterization of gene-derived SSR-markers in barley (Hordeum vulgare L.). Theor App/ Genet 2003, 106:411-422.

doi:10.1186/1471-2229-11-3

Cite this article as: Hsu et al:: An overview of the Phalaenopsis orchid genome through BAC end sequence analysis. BMC Plant Biology 2011 $11: 3$

\section{Submit your next manuscript to BioMed Central and take full advantage of:}

- Convenient online submission

- Thorough peer review

- No space constraints or color figure charges

- Immediate publication on acceptance

- Inclusion in PubMed, CAS, Scopus and Google Scholar

- Research which is freely available for redistribution

Submit your manuscript at www biomedcentral.com/submit
C Biomed Central 